\title{
Errata:
}

\section{Development of a Versatile Tensile Tester for Ultra-fine Fibers}

\author{
ENOMOTO Yuji a, b, *, TAKAYANAGI Ryota ${ }^{\text {a, c }}$, NAGASE Masachika ${ }^{\text {a, c }}$, NODA Yotaro ${ }^{\text {a, d }}$, \\ TAMAKI Yashuto ${ }^{\text {a }}$, KIM Ick Soo ${ }^{\text {a }}$, OKAWARA Yasuyuki ${ }^{\mathrm{e}}$, KAGA Yoshio ${ }^{\mathrm{e}}$, \\ OSAWA Yoshimasa ${ }^{\mathrm{e}}$, TAKAHASHI Toru ${ }^{\mathrm{e}}$
}

Journal of Textile Engineering (2011), Vol. 57, No. 1, 15-20

${ }^{a}$ Faculty of Textile Science and Technology, Shinshu University

$\mathrm{b}$ Toyama Industrial Technology Center

${ }^{\mathrm{c}}$ Seiko-Epson Co.

${ }^{\mathrm{d}} \mathrm{NEC}$ Co.

${ }^{\text {e }}$ Rhesca Co. Ltd.

The errors were found in the above paper. The corrections are given in the following table.

\begin{tabular}{|c|c|c|c|}
\hline Vol. & Page & Error & Correction \\
\hline 57 & 15 & $\begin{array}{l}\text { Key Words: Tensile test, Three-point } \\
\text { flexural test, Young's modulus, Breaking } \\
\text { strength, Stress relaxation, Nanofiber, } \\
\text { Young's modulus, Electrospinning }\end{array}$ & $\begin{array}{l}\text { Key Words: Fiber tension test, Three-point } \\
\text { flexural test, Stress relaxation test, } \\
\text { Electrospun Nanofiber }\end{array}$ \\
\hline 57 & 20 & $\begin{array}{l}\text { In our recent work }[10] \text {, however, thicker } \\
\text { metallic coating; } \mathrm{Cu} \text { film of } 50 \mathrm{~nm} \text { onto } \\
\text { polymeric single nanofibers led to increase } \\
\text { the tensile strength and Young's modulus } \\
{[10] \text {. }}\end{array}$ & $\begin{array}{l}\text { In our recent work }[13] \text {, however, thicker } \\
\text { metallic coating; } \mathrm{Cu} \text { film of } 50 \mathrm{~nm} \text { onto } \\
\text { polymeric single nanofibers led to increase } \\
\text { the tensile strength and Young's modulus. }\end{array}$ \\
\hline 57 & 20 & $\begin{array}{l}\text { [8] Enomoto Y, Kim IS, Kaga Y, } \\
\text { Okawara Y (2008) Japanese Patent } \\
\text { Application 2008-325656 } \\
\text { [9] KimIS, EnomotoY, TakahashiT (2009) } \\
\text { Sen'iGakkaishi, 65, } 325 \\
\text { [10] KimHR, BangHS, KimBS, } \\
\text { WatanabeY, EnomotoY, Kim IS (2010) } \\
\text { Tech-Connect World } 2010 \text { Proceedings } \\
\text { Vol. 2, 149-152 }\end{array}$ & $\begin{array}{l}\text { [11] Enomoto Y, Kim IS, Kaga Y, } \\
\text { Okawara Y (2008) Japanese Patent } \\
\text { Application 2008-325656 } \\
\text { [12] Kim IS, Enomoto Y, Takahashi T } \\
\text { (2009) Sen'i Gakkaishi, 65, } 325 \\
\text { [13] Kim HR, Bang HS, Kim BS, } \\
\text { Watanabe Y, Enomoto Y, Kim IS } \\
\text { (2010) Tech-Connect World } 2010 \\
\text { Proceedings Vol. 2, 149-152 }\end{array}$ \\
\hline
\end{tabular}

